\title{
Effect of Reducing Inflammation of Coptis chinensis Extract-Ceramide Complex through ECS Control in Atopic Dermatitis
}

\author{
Sang Hyun $\mathrm{Ahn}^{1}$, Ki Bong Kim${ }^{2,3 *}$ \\ ${ }^{1}$ Department of Anatomy, College of Korean Medicine, Semyung University \\ ${ }^{2}$ Department of Korean Pediatrics, Pusan National University Korean Hospital \\ ${ }^{3}$ School of Korean Medicine, Pusan National University
}

\begin{abstract}
Objectives: The purpose of this study was to confirm effect of reducing inflammation of Coptis chinensis extract -ceramide complex through the endocannabinoid system (ECS) control in atopic dermatitis.

Methods: 8-week-old ICR mice were divided into normal group (Ctrl), lipid barrier elimination group (ADE), palmitoylethanolamide treated group after lipid barrier elimination (PEAT), and Coptis chinensis extract-ceramide complex applied group after lipid barrier elimination (CRA). After inducing atopic dermatitis, cannabinoid receptor (CB) 1, CB2, CD68, p-I $\kappa \mathrm{B}$, iNOS, substance $\mathrm{P}$ and serotonin were observed to confirm the regulation of the ECS, macrophage activity and mast cell activity.

Results: $\mathrm{CB} 1$ and $\mathrm{CB} 2$ showed higher positive reactions in the CRA than in the ADE and PEAT. CD68, p-I $\kappa \mathrm{B}$ and iNOS showed higher positive reaction in the ADE, PEAT and CRA than in the Ctrl, but the increase in the positive reaction was lower in the CEA compared to the ADE and PEAT. Substance P and serotonin showed higher positive reaction in the ADE, PEAT and CRA than in the Ctrl, but the increase in the positive reaction was lower in the CEA compared to the ADE and PEAT.

Conclusions: The effects of Coptis chinensis extract -ceramide complex were confirmed on the regulation of the ECS, macrophage activity and mast cell activity.
\end{abstract}

$\overline{K e y}$ Words : Coptis chinensis, Atopic dermatitis, Ceramide, Endocannabinoid system (ECS), CB1, CB2

\section{Introduction}

Atopic dermatitis (AD) is a chronic inflammatory skin disease with a high prevalence in children ${ }^{1)}$. $\mathrm{AD}$ is caused by a combination of various causes. Patients with atopic dermatitis complain of symptoms such as erythema, edema, severe itching, and exudation $^{2)}$. Atopic dermatitis patients often have allergic rhinitis and asthma ${ }^{3)}$. These diseases progress chronically due to environmental changes due to industrialization, improper eating habits, and stress, and continue into adulthood ${ }^{4}$.

In atopic dermatitis, the skin is dry or itchy, and eczema erythematosus appears. These skin symptoms are due to damage to the skin barrier. When ceramide is reduced in the stratum

\footnotetext{
- Received : 7 October 2021

- Accepted : 8 November 2021

- Correspondence to : Kibong Kim

Department of Korean Pediatrics, Pusan National University Korean Hospital

Geumo-ro 20, Mulgeum-eup, Yangsan-si, Gyeongsangnam-do, 50612, Republic of Korea

Tel : +82-55-360-5952, Fax : +82-55-360-5952, E-mail : kkb@pusan.ac.kr
} 
corneum of the skin, the skin barrier function and moisture retention function that ceramide is responsible for decreases ${ }^{5}$. The causes of atopic dermatitis are diverse and complex. Physical and functional damage of the skin barrier occurs due to various causes, resulting in secondary immune system problems ${ }^{6}$.

In Korean traditional medicine, atopic dermatitis is caused by moist heat (濕熱) caused by congenital weakness, or by the intrusion of wind heat (風熱) from the outside and the accumulation of moist heat inside ${ }^{7)}$. Treatments includes 'dispel wind to clear heat (祛風清熱)' and 'nourish blood to moisten dryness (養血潤燥) ${ }^{38}$. Therefore, in the clinical field, herbs with a clear heat (清熱) effect are often used for the treatment of atopic dermatitis $^{9)}$.

Coptis chinensis (黃連) has medicinal effects of 'clear heat and dry dampness (清熱燥濕)' and 'purge fire and detoxify (瀉火解毒)', and also has antiviral and antibacterial effects ${ }^{10,11)}$. According to previous study ${ }^{12)}$, Coptis chinensis is also effective in suppressing inflammation and repairing skin damage from burns. Therefore, we tried to confirm the possibility of atopic dermatitis treatment by inhibiting skin damage and restoring the damaged skin barrier of Coptis chinensis extract-ceramide complex.

This study confirmed the regulation of the Endocannabinoid system (ECS) through cannabinoid receptor (CB) 1 and $\mathrm{CB} 2$. In addition, the regulation of macrophage activity through changes in Cluster of Differentiation 68 (CD68), phosphorylated inhibitor kappa $\mathrm{B}(\mathrm{p}-\mathrm{I} \kappa \mathrm{B})$, and inducible nitric oxide synthase (iNOS) and control of mast cell activity through changes in substance $\mathrm{P}$ and serotonin were confirmed. Through this, we report the possibility that Coptis chinensis extract-ceramide complex can alleviate the inflammatory response of atopic dermatitis.

\section{Materials and Methods}

\section{Materials}

1) Animals

As the experimental animals, 6-week-old male Balb/C mice $(20 \pm 1.5$ g, Jabio, Suwon, Korea) selected after acclimatization for 2 weeks in an aseptic breeding apparatus were used. They were divided into normal group (Ctrl), lipid barrier elimination group (ADE), palmitoylethanolamide (PEA) treated group after lipid barrier elimination (PEAT), and Coptis chinensis extract-ceramide complex applied group after lipid barrier elimination (CRA). 10 animals were assigned to each group. Animal experiments were conducted after approval from the Institutional Animal Care and Use Committee of Pusan University (IACUC No. PNU-2015-0924). The care and use of laboratory animals was conducted in accordance with NIH guidelines.

\section{2) Experimental drug}

Coptis chinensis (50 g, Omniherb, Daegu, Korea) was added in $100 \mathrm{ml}$ of distilled water, boiled for 3 hours, and then filtered. It was reduced to $50 \mathrm{ml}$ using a rotary evaporator, concentrated and freeze-dried to extract $8.2 \mathrm{~g}$ (yield: 16.4\%). This extract was diluted with physiological saline to make a 5\% extract dilution. This dilution was mixed with $5 \%$ ceramide $3 \mathrm{~B}$ (Ecofactory, Incheon, Korea) in a 1:1 ratio to 
make a 5\% Coptis chinensis extract-ceramide complex.

To CRA, $100 \mu \mathrm{l}$ of a $5 \%$ Coptis chinensis extract-ceramide complex was applied to the lipid barrier eliminated skin for 5 days. To PEAT, PEA (Sigma-Aldrich, St. Louis, MO) used as a control drug was dissolved in physiological saline $(100 \mu \mathrm{l})$ and then $10 \mathrm{mg} / \mathrm{kg}$ was orally administered for 5 days after eliminating the lipid barrier.

\section{Methods}

\section{1) lipid barrier elimination}

To eliminate the lipid barrier, the dorsal skin of the mouse was shaved, and then the stratum corneum desqumation was removed using Tape (3M, St. Paul, MN). $500 \mu 1$ of $10 \%$ sodium dodeecyl sulfate (SDS: Sigma-Aldrich, St. Louis, MO) was applied on the spot and then rubbed 30 times using a cotton swab to remove lipid lamella from the stratum corneum.

\section{2) Tissue section production}

Cardiac perfusion fixation was performed on the skin with vascular rinse and 10\% neutral buffered formalin (NBF). After the obtained dorsal skin was fixed in 10\% NBF for 24 hours, it was embedded in paraffin by a conventional method, and serial sections were made with a thickness of $5 \mu \mathrm{m}$.

\section{3) Immunohistochemical test}

Immunohistochemical staining was performed to investigate the immunohistological changes of the ECS, macrophage activity and mast cell activity. For immunohistochemical staining, antibodies such as anti-CB1, anti-CB2, anti-CD68, anti-p-IkB, anti-Substance $\mathrm{P}$ and anti-Serotonin were used.

First, the skin sections were subjected to proteolysis in proteinase $\mathrm{K}(20 \mu \mathrm{g} / \mathrm{ml}$, Agilent Dako, Santa Clara, CA) for 5 minutes and then treated with $10 \%$ normal goat serum (Vector Lab, Burlingame, CA) containing $1 \%$ fetal bovine serum (Sigma-Aldrich, St. Louis, MO) for 1 hour. Then, the primary antibody, mouse anti-CB1 (1:100, Santa Cruz Biotec, Dallas, TX), mouse anti-CB2 (1:50, Santa Cruz Biotec), mouse anti-CD68 (1:50, Santa Cruz Biotec), mouse anti-p-I $\kappa$ B (1:500, Abcam, Cambridge, UK), mouse anti-iNOS (1:250, Abcam), mouse anti-substans P (1:100, Santa Cruz Biotec) and mouse anti-serotonin (1:50, Santa Cruz Biotec) were reacted in a humidified chamber at $4{ }^{\circ} \mathrm{C}$ for 72 hours. The secondary antibody, biotinylated goat anti-mouse $\operatorname{IgG}$ (1:100, Abcam), was linked for 24 hours at room temperature, and reacted with avidin biotin complex kit (Vector Lab) for 1 hour at room temperature. After color development in $0.05 \mathrm{M}$ tris- $\mathrm{HCl}$ buffer $(\mathrm{pH}$ 7.4) containing $0.05 \% \quad 3,3$-diaminobenzidine and $0.01 \% \mathrm{HCl}$, counterstaining was performed with hematoxylin.

\section{4) Image analysis}

The results of immunohistochemistry were quantified (means \pm standard error) through image analysis using image Pro 10 (Media cybernetics, Rockville, MD). After randomly selecting 10 skin samples from each group, they were photographed at x400 magnification, and then images were analyzed with positive pixels (intensity 80-100) /20,000,000 pixels. 


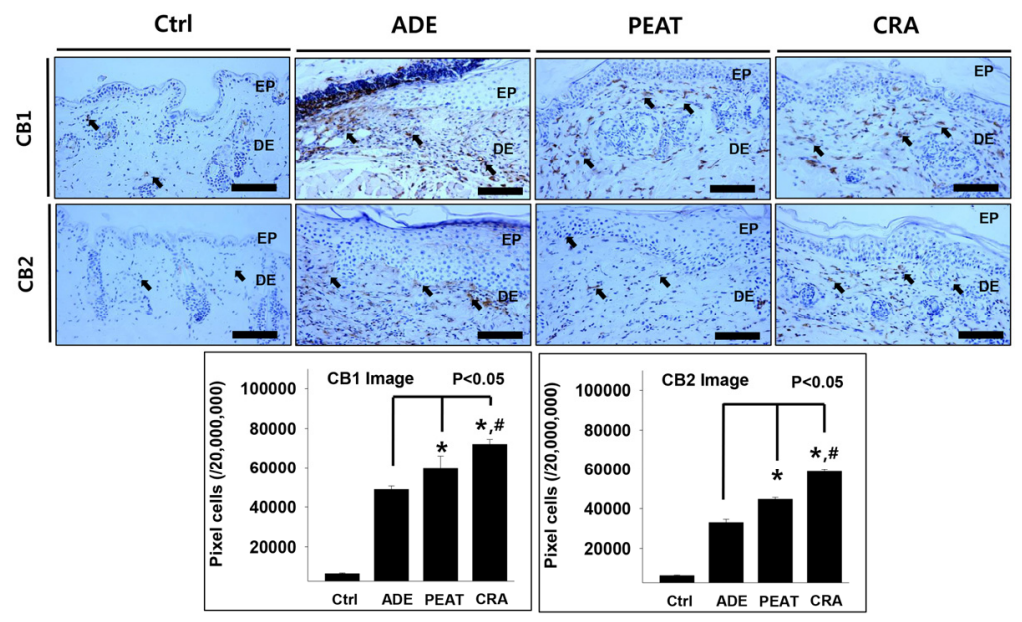

Fig 1. The alleviation of ECS in skin barrier by application of Coptis chinensis extract-ceramide complex.

CB1 and CB2 showed higher positive reactions in the CRA than in the ADE and PEAT. The expression of CB1 and CB2 (arrow indicates light brown particle) was significantly increased in the PEAT and CRA as compared with the ADE, the data of image analysis showed the same results. ${ }^{*}, p<0.05$ compared with ADE; \#, $p$ < 0.05 compared with PEAT. Ctrl, normal group; ADE, lipid barrier elimination group; PEAT, palmitoylethanolamide (PEA) treated group after lipid barrier elimination; CRA, Coptis chinensis extract-ceramide complex applied group after lipid barrier elimination; EP, epithelium; DE, dermis; bar size, 50um.

\section{5) Statistics}

For statistics, SPSS software (SPSS 25, SPSS Inc., Chicago, IL) was used. Significance ( $p$ $<0.05)$ was verified through one-way ANOVA, and Tukey HSD was performed for post hoc verification.

\section{Results}

\section{ECS regulation}

CB1 positive reaction was increased in the ADE, PEAT, and CRA compared to the Ctrl $(6,730 \pm 390 / 20,000,000$ pixel). The ADE $(48,832$ $\pm 1,716 / 20,000,000$ pixel) increased by $626 \%$ compared to the Ctrl. The PEAT $(59,878 \pm 6,161$ $120,000,000$ pixel) increased by $790 \%$ compared to the Ctrl, and the CRA $(72,024 \pm 2,471$ $/ 20,000,000$ pixel) increased by $970 \%$ compared to the Ctrl. The CRA had significantly a $48 \%$ higher positive reaction than the ADE and 20\% than the PEAT (Fig. 1).

CB2 positive reaction was increased in the ADE, PEAT, and CRA compared to the Ctrl $(6,583 \pm 274 / 20,000,000$ pixel). The ADE $(32,986$ $\pm 1,653 / 20,000,000$ pixel) increased by $401 \%$ compared to the Ctrl. The PEAT $(44,704 \pm 965$ $/ 20,000,000$ pixel) increased by $579 \%$ compared to the Ctrl, and the CRA $(58,739 \pm 992$ $/ 20,000,000$ pixel) increased by $792 \%$ compared to the Ctrl. The CRA had significantly a $78 \%$ higher positive reaction than the ADE and 31\% than the PEAT (Fig. 1).

\section{Macrophage activity regulation}

CD68 positive reaction was increased in the ADE, PEAT, and CRA compared to the Ctrl (7.508 $\pm 305 / 20,000,000$ pixel). The $\operatorname{ADE}(81,251$ $\pm 2,505 / 20,000,000$ pixel) increased by $982 \%$ 


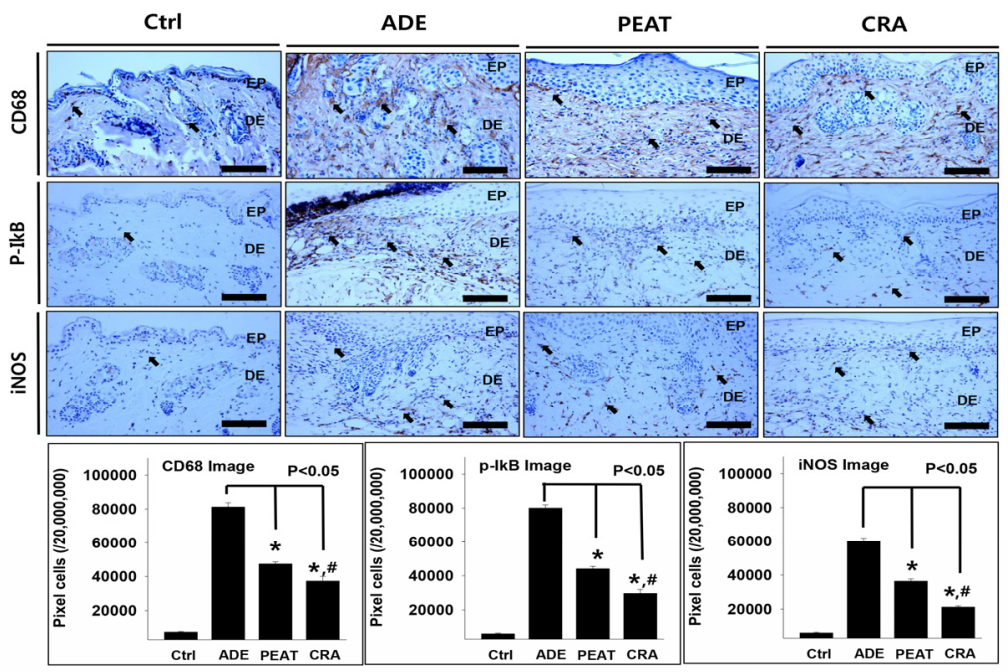

Fig 2. The regulation of macrophage activity in skin by application of Coptis chinensis extract-ceramide complex. CD68, $p-1 K B$ and iNOS (arrow indicates light brown particle) showed relatively higher positive reaction in the ADE, PEAT and CRA than in the Ctrl, but the increase in the positive reaction was lower in the CEA compared to the ADE and PEAT. ${ }^{*}, p<0.05$ compared with ADE; \#, $p$ $<0.05$ compared with PEAT. abbrevation same as Fig. 1.

compared to the Ctrl. The PEAT $(47,419 \pm$ $1,205 / 20,000,000$ pixel) increased by $532 \%$ compared to the Ctrl, and the CRA $(37,354 \pm$ $2,672 / 20,000,000$ pixel) increased by $398 \%$ compared to the Ctrl. The CRA had significantly a 54\% lower positive reaction than the $\mathrm{ADE}$ and $21 \%$ than the PEAT (Fig. 2).

$\mathrm{p}-\mathrm{I} \kappa \mathrm{B}$ positive reaction was increased in the ADE, PEAT, and CRA compared to the Ctrl $(6,147 \pm 370 / 20,000,000$ pixel). The $\operatorname{ADE}(80,073$ $\pm 1,889 / 20,000,000$ pixel) increased by $1,203 \%$ compared to the Ctrl. The PEAT $(44,090 \pm 1,429$ $120,000,000$ pixel) increased by $617 \%$ compared to the Ctrl, and the CRA $(29,703 \pm 2,351$ $120,000,000$ pixel) increased by $383 \%$ compared to the Ctrl. The CRA had significantly a $63 \%$ lower positive reaction than the $\mathrm{ADE}$ and $33 \%$ than the PEAT (Fig. 2).

iNOS positive reaction was increased in the
ADE, PEAT, and CRA compared to the Ctrl $(6,068 \pm 420 / 20,000,000$ pixel). The $\operatorname{ADE}(60,145$ $\pm 1,701 / 20,000,000$ pixel) increased by $891 \%$ compared to the Ctrl. The PEAT $(36,406 \pm 1,335$ $/ 20,000,000$ pixel) increased by $500 \%$ compared to the Ctrl, and the CRA $(21,240 \pm 673 / 20,000,000$ pixel) increased by $250 \%$ compared to the Ctrl. The CRA had significantly a $65 \%$ lower positive reaction than the $\mathrm{ADE}$ and $42 \%$ than the PEAT (Fig. 2).

\section{Mast cell activity regulation}

Substance $\mathrm{P}$ positive reaction was increased in the ADE, PEAT, and CRA compared to the Ctrl $(5,088 \pm 246 / 20,000,000$ pixel). The $\operatorname{ADE}(74,641$ $\pm 1,663 / 20,000,000$ pixel) increased by $1367 \%$ compared to the Ctrl. The PEAT $(57,047 \pm 1,861$ $/ 20,000,000$ pixel) increased by $1,021 \%$ compared to the Ctrl, and the CRA $(25,610 \pm 854 / 20,000,000$ 


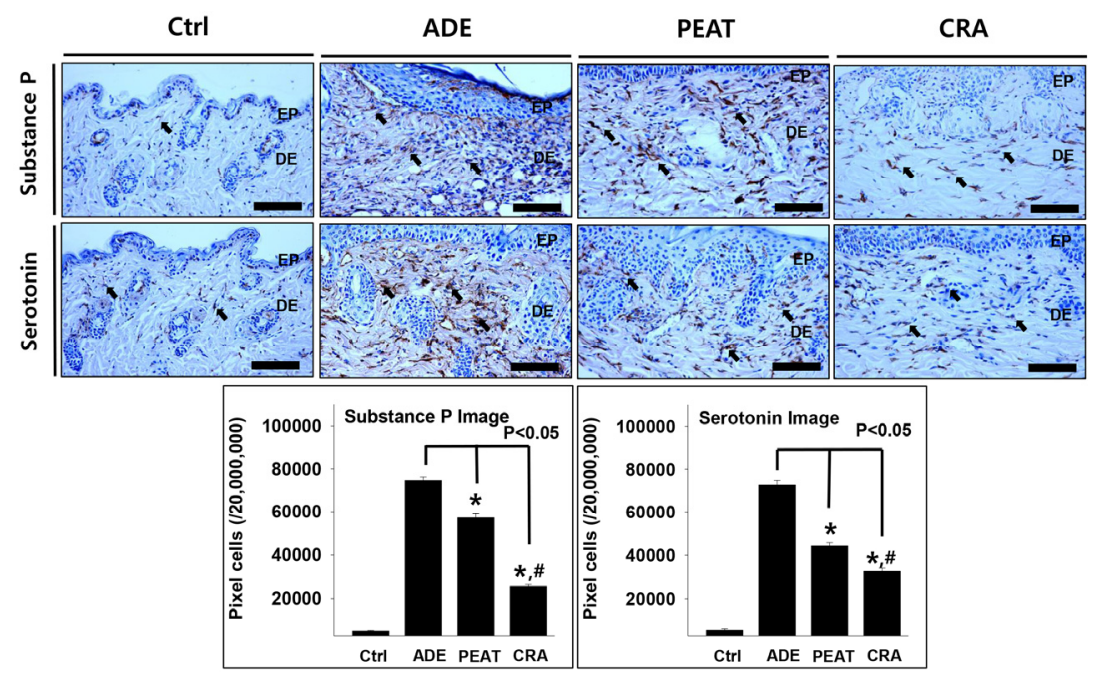

Fig. 3. The regulation of mast cell in skin by application of Coptis chinensis extract-ceramide complex.

The positive reaction of Substance $\mathrm{P}$ and serotonin (arrow indicates light brown particle) is higher in the ADE, PEAT and CRA than in the Ctrl, but the increase in the positive reaction was lower in the CEA compared to the ADE and PEAT. ${ }^{*}, p<0.05$ compared with ADE; \#, $p<0.05$ compared with PEAT. abbrevation same as Fig. 1.

pixel) increased by $403 \%$ compared to the Ctrl. The CRA had significantly a $66 \%$ lower positive reaction than the $\mathrm{ADE}$ and $55 \%$ than the PEAT (Fig. 3).

Serotonin positive reaction was increased in the ADE, PEAT, and CRA compared to the Ctrl $(5,508 \pm 668 / 20,000,000$ pixel). The $\operatorname{ADE}(72,852$ $\pm 1,981 / 20,000,000$ pixel) increased by $1,223 \%$ compared to the Ctrl. The PEAT $(44,253 \pm 1,493$ $/ 20,000,000$ pixel) increased by $703 \%$ compared to the Ctrl, and the CRA $(32,592 \pm 1,500$ /20,000,000 pixel) increased by $492 \%$ compared to the Ctrl. The CRA had significantly a $55 \%$ lower positive reaction than the $\mathrm{ADE}$ and 26\% than the PEAT (Fig. 3).

\section{Discussion}

Atopic dermatitis is a chronic disease that deteriorates the quality of life due to recurrence and worsening of symptoms at various ages. There are various causes of atopic dermatitis, and at present, there is no fundamental treatment, only to relieve symptoms or prevent further deterioration. Currently, antihistamines and steroids are used for the treatment of atopic dermatitis, but it is only a temporary symptom relief rather than a fundamental treatment ${ }^{13)}$.

Coptis chinensis extract-ceramide complex suppresses inflammation and restores skin damage with its cleansing heat and detoxifying effect. ${ }^{10-12)}$. In this study, we confirmed the possibility that Coptis chinensis extract-ceramide complex could be a therapeutic agent for atopic dermatitis by inhibiting skin damage and restoring damaged skin barrier.

The ECS is a biological system that regulates appetite, pain sensation, mood, and memory. The 
ECS consists of cannabinoid receptors (CBRs), endogenous ligands of CBRs, and enzymes involved in the synthesis and degradation of $\mathrm{CB}^{14)}$. Increased or decreased the ECS has been associated with various pathological conditions ${ }^{15}$. Transient changes in ECS action can reduce or slow the progression of various bodily compensatory responses or diseases. On the other hand, the activity of the ECS may reflect bodily defects or act pathogenic ${ }^{16,17)}$. In particular, the ECS is involved in various responses of skin cells ${ }^{18)}$. Activation of CB1 and CB2 in epidermal cells inhibits keratinocyte proliferation and differentiation, induces inflammation, and induces apoptosis ${ }^{19-21)}$. Various CBs suppress sensory symptoms such as pain and itching through $\mathrm{CB}^{22,23)}$. The ECS regulates immune and inflammatory responses as well as cell differentiation in the skin. Therefore, even fine adjustment of the ECS can promote or alleviate skin diseases such as atopic dermatitis ${ }^{24)}$. For this reason, research using the ECS as a new treatment for skin diseases is emerging ${ }^{25)}$.

In this study, both PEAT and CRA increased significantly in $\mathrm{CB} 1$ and $\mathrm{CB} 2$ than $\mathrm{ADE}$, and CRA increased more than PEAT. The results of this study indicate that Coptis chinensis extract -ceramide complex produces the ECS components such as $\mathrm{CB} 1$ and $\mathrm{CB} 2$. This suggests the possibility of alleviating the symptoms of atopic dermatitis through the intervention of the Coptis chinensis extract-ceramide complex in the action of the ECS.

Mitogen-activated protein kinases (MAPKs), including extracellular signal-regulated kinase (ERK) and c-Jun N-terminal kinases (JNK), regulate the inflammatory response through nuclear factor kappa B (NF- $\kappa \mathrm{B})$ activation $^{26)}$. NF-kB is a key regulator in the cellular inflammatory response and exists in an inactive state in the cytoplasm in a state of binding with inhibitor of $\mathrm{NF}-\mathrm{kB}(\mathrm{IkB})^{27)}$. When a cell is damaged or stressed, NF-kB is separated into IkB by IkB kinase (IKK), and enters the nucleus to cause an inflammatory response ${ }^{28)}$. And iNOS, which is transcribed by the pro-inflammatory enzyme produced by NF-kB, excessively produces nitric oxide (NO) to create oxidative stress, thus causing tissue damage and inflammation ${ }^{29}$.

In this study, both PEAT and CRA decreased significantly in $\mathrm{CD} 68, \mathrm{p}-\mathrm{I} \kappa \mathrm{B}$, and iNOS than ADE, and CRA decreased more than PEAT. This means that the Coptis chinensis extract-ceramide complex is likely to be involved in the regulation of macrophage activity.

Symptoms of atopic dermatitis, such as severe pruritus, are initiated by immunoglobulin E (IgE) -mediated degranulation of mast cells ${ }^{30)}$. When $\mathrm{IgE}$ and antigen bind to receptors expressed on the surface of mast cells, mast cells are activated, and the activated mast cells secrete inflammatory mediators such as susbstance $\mathrm{P}$ and Matrix metallopeptidase (MMP) -9 to induce an inflammatory response. Substance $\mathrm{P}$ not only regulates the secretion of various cytokines, but also participates in the inflammatory response and induces itching through vascular relaxation, increased vascular permeability, and histamin secretion. So, Substance P can be said to be an indicator of itching symptoms. Serotonin exists in platelets, activates dermal mast cells, secretes histamine, and acts on 5-HT3 receptors in the central nervous system to induce pruritus ${ }^{31}$. 
In this study, substance $\mathrm{P}$ and serotonin showed higher positive reaction in the ADE, PEAT and CRA than in the Ctrl, but the increase in the positive reaction was lower in the CEA compared to the ADE and PEAT. This result means that the Coptis chinensis extract-ceramide complex can modulate mast cell activity. This showes the possibility that the Coptis chinensis extract -ceramide complex can alleviate symptoms of atopic dermatitis such as itching.

Coptis chinensis extract-ceramide complex inhibited the pathological symptoms of the skin barrier and skin damage in atopic dermatitis. In addition, it promoted the formation of the ECS components such as $\mathrm{CB} 1$ and $\mathrm{CB} 2$ and a lipid barrier, and inhibited proliferation and invasion of inflammatory cells.

These results show that Coptis chinensis extract -ceramide complex maintains skin homeostasis, improves skin barrier formation and suppresses inflammation through the ECS intervention. Therefore, Coptis chinensis extract-ceramide complex is expected to alleviate the symptoms of atopic dermatitis. However, since this study was limited to animal experiments, additional clinical studies are needed in the future.

\section{Conclusion}

In this study, the effects of Coptis chinensis extract-ceramide complex were confirmed on the regulation of the ECS through $\mathrm{CB} 1$ and $\mathrm{CB} 2$, the regulation of macrophage activity through CD68, $\mathrm{p}-\mathrm{I} \kappa \mathrm{B}$, and iNOS, and the regulation of mast cell activity through substance $\mathrm{P}$ and serotonin.

1. $\mathrm{CB} 1$ and $\mathrm{CB} 2$ showed higher positive reactions in the CRA than in the ADE and PEAT.

2. CD68, p-I $\kappa \mathrm{B}$ and iNOS showed higher positive reaction in the ADE, PEAT and CRA than in the Ctrl, but the increase in the positive reaction was lower in the CEA compared to the ADE and PEAT.

3. Substance $P$ and serotonin showed higher positive reaction in the $\mathrm{ADE}, \mathrm{PEAT}$ and $\mathrm{CRA}$ than in the Ctrl, but the increase in the positive reaction was lower in the CEA compared to the ADE and PEAT.

\section{Acknowledgement}

This work was supported by a 2-Year Research Grant of Pusan National University, Busan, Republic of Korea.

\section{References}

1. Kim, J.H., (2004). Current understanding of atopic dermatitis. Pediatr Allergy Respir Dis, 14(1), 12-23.

2. Blume-Peytavi, U., \& Metz, M. (2012). Atopic dermatitis in children: management of pruritus. J Eur Acad Dermatol Venereol, 26, 2-8. https://doi.org/10.1111/j.1468-3083.2012.04710.x

3. Lee, J.Y., \& Kim, D.G. (1999). The Clinical Study of Atopic Dermatitis. J Pediatr Korean Med, 13(2), 171-86.

4. Jee, H.M., Kim, K.W., Kim, C.S., Sohn, M.H., Shin, D.C., \& Kim, K.E. (2009). Prevalence of asthma, rhinitis and eczema in Korean children using the International Study of Asthma and Allergies in Childhood (ISAAC) questionnaires. Pediatr Allergy Respir Dis, 
$19(2), 165-72$.

5. Kim, B.E., \& Leung, D. (2012). Epidermal barrier in atopic dermatitis. Immunol Res, 4(1), 12-6. http://dx.doi.org/10.4168/aair.2012.

\subsubsection{2}

6. Hogan, M.B., Peele, K., \& Wilson, N.W. (2012). Skin barrier function and its importance at the start of the atopic march. J Allergy, 901940 . https://doi.org/10.1155/2012/901940

7. Ahn, K.M., Kim, J.H., Kwon, H.J., Chae, Y.M., Hahm, M.I., Lee, K.J., et al. (2011). The prevalence of symptoms of asthma, allergic rhinoconjunctivitis, and eczema in Korean children: nationwide cross-sectional survey using complex sampling design. J Korean Med Assoc, 54(7), 769-78. https://doi.org/ 10.5124/jkma.2011.54.7.769

8. Kim, M.J., \& Lee, S.Y. (2000). A Literature Study of Atopic Dermatitis for Children. J Pediatr Korean Med, 14(2), 167-82.

9. Muluye, R.A., Bian, Y., \& Alemu, P.N. (2014). Anti-inflammatory and antimicrobial effects of heat-clearing Chinese herbs: a current review. J Tradit Complement Med, 4(2), 93-8. https://doi.org/10.4103/2225-4110.126635

10. Chin, L.W., Cheng, Y.W., Lin, S.S., Lai, Y.Y., Lin, L.Y., Chou, M.Y., et al. (2010). Anti-herpes simplex virus effects of berberine from Coptidis Rhizoma, a major component of a Chinese herbal medicine, Ching-Wei-San. Arch Virol, 155(12), 1933-41. https://doi.org/ 10.1007/s00705-010-0779-9

11. Yu, Y.E., Park, E.Y., Jung, D.H., Byun, S.H., Kim, S.C., \& Park, S.M. (2010). Antibacterial activity of oriental medicinal herb extracts against skin pathogens. J Life Sci, 20(7),
1143-50. https://doi.org/10.5352/JLS.2010.20. 7.1143

12. Kim, H.K., \& Hong, S.U. (2011). The anti-inflammatory effects of Huang-Lyun (Coptidis Rhizoma, CR) on injured tissue after burn elicitation. J Korean Oriental Med, 32(2), 1-13.

13. Baron, E.D., Barzilai, D., Johnston, G., Kawashima, M., Takigawa, M., Nakagawa, H., et al. (2002). Stevens SR. Atopic dermatitis management: comparing the treatment patterns of dermatologists in Japan, U.S.A. and U.K. Br J Dermatol, 147, 710-5. https://doi.org/10. 1046/j.1365-2133.2002.04895.x

14. Salzet, M. (2000). Invertebrate molecular neuroimmune processes. Brain Res Rev, 34(1), 69-79. https://doi.org/10.1016/S0165 $-0173(00) 00041-2$

15. Pacher, P., Batkai, S., \& Kunos, G. (2006). The endocannabinoid system as an emerging target of pharmacotherapy. Pharmacol Rev, 58(3), 389-462. https://doi.org/10.1124/pr.58.3.2

16. Kupczyk, P., Reich, A., \& Szepietowski, J.C. (2009). Cannabinoid system in the skin - a possible target for future therapies in dermatology. Exp Dermatol, 18(8), 669-79. https://doi.org/10.1111/j.1600-0625.2009.00923.x

17. Miller, L.K., \& Devi, L.A. (2011). The highs and lows of cannabinoid receptor expression in disease: mechanisms and their therapeutic implications. Pharmacol Rev, 63(3), 461-70. https://doi.org/10.1124/pr.110.003491

18. Luger, T.A. (2002). Neuromediators--a crucial component of the skin immune system. J Dermatol Sci, 30(2), 87-93. https://doi.org/10. 1016/S0923-1811(02)00103-2 
19. Maccarrone, M., Rienzo, M., Battista, N., Gasperi, V., Guerrieri, P., Rossi, A., et al. (2003). The Endocannabinoid System in Human Keratinocytes: Evidence that anandamide inhibits epidermal differentiation through CB1 receptor-dependent inhibition of protein kinase $\mathrm{C}$, activation protein-1, and transglutaminase. J Biol Chem, 278(36), 33896-903. https://doi.org/ 10.1074/jbc.M303994200

20. Karsak, M., Gaffal, E., Date, R., Wang -Eckhardt, L., Rehnelt, J., \& Petrosino, S. (2007). Attenuation of Allergic Contact Dermatitis Through the Endocannabinoid System. Science, 316(5830), 1494-97. https://doi.org/10.1126/ science. 1142265

21. Casanova, M.L., Blazquez, C., Martinez-Palacio, J., Villanueva, C., Fernandez-Acenero, M.J., Huffman, J.W., et al. (2003). Inhibition of skin tumor growth and angiogenesis in vivo by activation of cannabinoid receptors. J Clin Invest, 111(1), 43-50. https://doi.org/10.1172/ JCI16116.

22. Walker, J.M., \& Hohmann, A.G. (2005). Cannabinoid mechanisms of pain suppression. Handb Exp Pharmacol, 168, 509-54. https://doi.org/10.1007/3-540-26573-2_17

23. Jhaveri, M.D., Richardson, D., \& Chapman, V. (2007). Endocannabinoid metabolism and uptake: novel targets for neuropathic and inflammatory pain. Br J Pharmacol, 152(5), 624-32. https://dx.doi.org/10.1038\%2Fsj.bjp.07 07433

24. Tóth, B.I., Dobrosi, N., Dajnoki, A., Czifra, G., Olah, A., Szollosi, A.G., et al. (2011). Endocannabinoids Modulate Human Epidermal Keratinocyte Proliferation and Survival via the Sequential Engagement of Cannabinoid Receptor-1 and Transient Receptor Potential Vanilloid-1. J Invest Dermatol, 131(5), 1095-104. https://doi.org/10.1038/jid.2010.421

25. Tóth, K.F., Ádám, D., Bíró, T., \& Olah, A. (2019). Cannabinoid Signaling in the Skin: Therapeutic Potential of the "C(ut)annabinoid" System. Molecules, 24(5), 918. https://doi.org/ 10.3390/molecules 24050918

26. Arbabi, S., \& Maier, R.V. (2002). Mitogen -activated protein kinases. Crit Care Med, 30(1), 74-9.

27. Chung, H.Y., Cesari, M., Anton, S., Marzetti, E., Giovannini, S., Seo, A.Y., et al. (2009). Molecular inflammation: underpinnings of aging and age-related diseases. Ageing Res Rev, 8(1), 18-30. https://doi.org/10.1016/j.arr. 2008.07.002

28. Baeuerle, P.A., \& Baltimore, D. (1996). NF-kappa B: ten years after. Cell, 87(1), 13-20. https://doi.org/10.1016/s0092-8674(00) 81318-5

29. Aktan, F. (2004). iNOS-mediated nitric oxide production and its regulation. Life Sciences, 75(6), 639-53. https://doi.org/10.1016/j.lfs.2003. 10.042

30. Kay, A.B.(2001). Allergy and allergic diseases. First of two parts. N Engl J Med, 344, 30-7. https://doi.org/10.1056/nejm200101043440106

31. Jo, J.W., \& Kim, C.Y. (2017). The Neurotransmitter Pathway of Itching. J Life Sci, 27(5), 600-10. https://doi.org/10.5352/JLS.2017 


\section{ORCID}

Sang Hyun Ahn https://orcid.org/0000-0002-1526-4397

Ki Bong Kim https://orcid.org/0000-0002-0096-5836 